\title{
Kinetic theory of shot noise in nondegenerate diffusive conductors
}

\author{
$\mathrm{H}$ Schomerus \\ Instituut-Lorentz, Untversıtelt Leıden PO Box 9506, 2300 RA Letden, The Netherlands \\ E G Mishchenko \\ Instituut-Loientz, Untversutet Letden, PO Box 9506, 2300 RA Leiden, The Netherlands \\ and $L D$ Landau Institute for Theoretcal Phystcs, Kosygun 2, Moscow 117334, Russta \\ C W J Beenakker \\ Instituut-Lorentz, Untversitelt Letden, PO Box 9506, 2300 RA Letden, The Netherlands
}

(Received 29 January 1999)

\begin{abstract}
We investigate current fluctuations in nondegenerate semiconductors, on length scales intermediate between the elastic and inelastic mean free paths We present an exact solution of the nonlinear kinetic equations in the regime of space-charge Imited conduction, without resortmg to the drift approximation of previous work By including the effects of a finite voltage and carrier density in the contact region, a quantitative agreement is obtained with Monte Carlo simulations by Gonzalez et al, for a model of an energy-independent elastic scattering rate The shot-noise power $P$ is suppressed below the Poisson value $P_{\text {Poissor }}=2 e \bar{I}$ (at mean current $\bar{I})$ by the Coulomb repulsion of the carriers The exact suppression factor is close to $1 / 3$ in a three-dimensional system, in agreement with the simulations and with the drift approximation Including an energy dependence of the scattering rate has a small effect on the suppression factor for the case of short-range scattering by uncharged impuritıes or quasıelastic scatterıng by acoustıc phonons Long-range scatterıng by charged impurities remains an open problem [S0163-1829(99)02931-8]
\end{abstract}

\section{INTRODUCTION}

The kinetic theory of nonequilibilum fluctuations in an electron gas was pioneered by Kadomtsev in 1957 (Ref. 1) and fully developed ten years late ${ }^{23}$ The theory has been comprehensively reviewed by Kogan ${ }^{4}$ In recent years there has been a revival of interest in this field because of the discovery of fundamental effects on the mesoscopic length scale (See Ref 5 for a recent review) One of these effects is the sub-Porssonian shot noise in degenerate electron gases on length scales intermediate between the mean free path $l$ for elastic impunity scattering and the inelastic mean fiee path $l_{1 \mathrm{n}}$ for electron-phonon or electron-election scattering The unversal one-thind suppression of the shot-noise powei predicted theoretically ${ }^{67}$ has been observed in experiments on semiconductor or metal wires of micrometer length ${ }^{8-11}$

The electron density in these experiments is sufficiently high that the election gas is degenerate The reduction of the shot-noise power

$$
P=2 \int_{-\infty}^{\infty} d t^{\prime} \overline{\delta I(t) \delta I\left(t+t^{\prime}\right)}
$$

[with $\delta I(t)$ being the fluctuations of the cunent around the mean current $\bar{I}]$ below the Poisson value $P_{\text {Poisson }}=2 e \bar{I}$ is then the result of conelations induced by the Paul exclusion principle. When the electron density is reduced, the Pauli principle becomes ineffective One enters then the regime of a nondegenerate election gas, studied recently in Monte Carlo simulations by González et al ${ }^{12}$ In a model of eneı gyindependent three-dimensional elastıc impurity scatteing, these authois found the very same ratio $P / P_{\text {Poisson }}=1 / 3$ as 1 n the degenerate case The origin of the suppression is quite different, however, being due to corielations induced by long-range Coulomb repulsion-rather than by the Paul principle The one-third suppression of shot noise in the computer simulations 1equired a large voltage and short scieening length, but was found to be otherwise independent of material parameters

Subsequent analytical work by one of the authors ${ }^{13}$ explained this universality as a feature of the regime of spacecharge limited conduction The kinetic equations in this iegime are highly nonlınear and could only be solved in the approximation that the diffusion teim is neglected compared to the drift term This is a questionable appioximation. The ratio of the two teims $1 \mathrm{~s} 1 / d$, with $d$ the dimensionality of the density of states The result of Ref 13 ,

$$
P / P_{\text {Polsson }}=\frac{12}{5} \frac{3 d^{2}+22 d+64}{(d+2)(3 d+4)(3 d+8)}
$$

becomes exact in the large- $d$ limit, when $P / P_{\text {Porsson }} \rightarrow 4 / 5 d$, but has an erroi of unknown magnitude for the physically relevant value $d=3$.

The main purpose of the present pape1 is to report the exact solution of the kinetic equations in the space-charge limited ti ansport regime We find that inclusion of the diffusion term has a pronounced effect on the spatial dependence of the electic field, although the ultimate effect on the norse power tuins out to be relatively small The exact suppression factor differs from Eq (12) by about $10 \%$ for $d=3$ We find 


$$
P / P_{\text {Poisson }}= \begin{cases}06857 & \text { for } d=1 \\ 04440 & \text { for } d=2 \\ 03097 & \text { for } d=3,\end{cases}
$$

close to the values reported by González et al (although their surmise that $P / P_{\text {Poisson }}$ is a simple fraction $1 / d$ for $d$ $=2,3$ is not borne out by this exact calculation) By including the effects of a finite temperature and scieening length, we obtain excellent agreement with the electric-field profiles in the simulations (which could not be achieved in the diff approximation of Ref 13) and determine the conditions for space-charge limited conduction We also go beyond prev1ous work by calculating to what extent the shot-norse suppression factor varies with the energy dependence of the scattering rate (This breakdown of universality was antic1pated in Refs 13 and 14)

The paper is organized as follows The kinetic theory is introduced in Sec II, where we summarize the basic equations and emphasize the differences with the degenerate case In Sec III we formulate the problem for the regime of spacecharge limited conduction In Sec IV we solve the kinetic equations for the case of an energy-independent scattering rate and compare with the Monte Carlo simulations ${ }^{12} \mathrm{We}$ study separately the capacitance fluctuations The effect of deviations from the conditions of space-charge limited con duction is also investigated Energy dependence in the scattering rate is considered in Sec V We conclude in Sec VI with a discussion of the experumental observability in connection with electron-phonon scatteing

\section{KINETIC THEORY}

\section{A. Boltzmann-Langevin equation}

Our staiting point is the same kinetic theory ${ }^{1-4}$ used to study shot noise in degenerate conductors ${ }^{5715-21}$ We summarize the basic equations, emphasizing the differences in the nondegenerate case The density $f(\mathbf{r}, \mathbf{p}, t)$ of carries at position $\mathbf{r}$ and momentum $\mathbf{p}=m \mathbf{v}$ at time $t$ (where $m$ is the effective mass and $\mathbf{v}$ is the velocity) satisfies the BoltzmannLangevin equation

$$
\left[\frac{\partial}{\partial t}+\mathbf{v} \frac{\partial}{\partial \mathbf{r}}+e \mathbf{E}(\mathbf{r}, t) \frac{\partial}{\partial \mathbf{p}}\right] f(\mathbf{r}, \mathbf{p}, t)=\mathcal{S}+\delta J
$$

Here $\mathbf{E}(\mathbf{r}, t)$ is the electric field (we take the charge of the caniers positive), $\mathcal{S}(\mathbf{r}, \mathbf{p}, t)$ is the collision integral, and $\delta J(\mathbf{r}, \mathbf{p}, t)$ is a fluctuatıng source (or "Langevin curient") The collision integral desciubes the average effect of elastic impurity scattering,

$$
\mathcal{S}(\mathbf{r}, p \hat{\mathbf{n}}, t)=\int \frac{d \hat{\mathbf{n}}^{\prime}}{\Omega} W_{\varepsilon}\left(\hat{\mathbf{n}} \hat{\mathbf{n}}^{\prime}\right)\left[f\left(\mathbf{r}, p \hat{\mathbf{n}}^{\prime}, t\right)-f(\mathbf{r}, p \hat{\mathbf{n}}, t)\right]
$$

The integial over the diuection $\hat{\mathbf{n}}=\mathbf{p} / p$ of the momentum extends over the surface of the unit spheie in $d$ dimensions, with surface area $\Omega=2 \pi^{d / 2} / \Gamma\left(\frac{1}{2} d\right)$ The scattering rate $W_{\varepsilon}\left(\hat{\mathbf{n}} \hat{\mathbf{n}}^{\prime}\right)$ depends on the kinetic energy $\varepsilon=p^{2} / 2 m$ and on the scattering angle $\hat{\mathbf{n}} \hat{\mathbf{n}}^{\prime}$ The effective mass $m$ is assumed to be energy independent
The stochastic Langevin current $\delta J$ vanishes on average, $\overline{\delta J}=0$, and has correlator ${ }^{2}$

$$
\begin{aligned}
& \overline{\delta J(\mathbf{r}, \mathbf{p}, t) \delta J\left(\mathbf{r}^{\prime}, \mathbf{p}^{\prime}, t^{\prime}\right)} \\
& =\delta\left(\mathbf{r}-\mathbf{r}^{\prime}\right) \delta\left(t-t^{\prime}\right) \delta\left(\varepsilon-\varepsilon^{\prime}\right) \frac{1}{\nu(\varepsilon)} \\
& \times\left[\delta\left(\hat{\mathbf{n}}-\hat{\mathbf{n}}^{\prime}\right) \int d \hat{\mathbf{n}}^{\prime \prime} W_{\varepsilon}\left(\hat{\mathbf{n}} \hat{\mathbf{n}}^{\prime \prime}\right)\left(\bar{f}+\bar{f}^{\prime \prime}-2 \bar{f} \bar{f}^{\prime \prime}\right)\right. \\
& \left.-W_{\varepsilon}\left(\hat{\mathbf{n}} \hat{\mathbf{n}}^{\prime}\right)\left(\bar{f}+\bar{f}^{\prime}-2 \bar{f} \bar{f}^{\prime}\right)\right],
\end{aligned}
$$

determined by the mean occupation number $\bar{f}$ [We abbiev1ated $\bar{f}^{\prime}=\bar{f}\left(\mathbf{r}, \mathbf{p}^{\prime}, t\right)$ and analogously for $\left.\bar{f}^{\prime \prime}\right]$ The density of states in $d$ dimensions is $\nu(\varepsilon)=m \Omega(2 m \varepsilon)^{d / 2-1}$, where we set Planck's constant $h \equiv 1$

A nondegenerate electron gas is characte1ized by $\bar{f} \ll 1$ In contrast to the degenerate case, the Pauli exclusion pinciple is then of no effect One consequence is that we may omit the terms quadiatic in $\bar{f}$ in the correlator (23) A second consequence is that deviations from equilibrium are no longer restricted to a nariow energy lange around the Felmı level, but extend over a bioad range of $\varepsilon$ One cannot, therefore, elimmate $\varepsilon$ as an independent valiable from the outset, as in the degenerate case

\section{B. Diffusion approximation}

We assume that the elastic mean free path is shoit compared to the dimensions of the conductor, so that we can make the diffusion approximation This consists in keeping only the two leading terms,

$$
f(\mathbf{r}, \hat{\mathbf{n}} \sqrt{2 m \varepsilon}, t)=\mathcal{F}(\mathbf{r}, \varepsilon, t)+\hat{\mathbf{n}} \mathbf{f}(\mathbf{r}, \varepsilon, t),
$$

of a multipole expansion in the momentum direction $\hat{\mathbf{n}}$ We substitute Eq (24) into the Boltzmann-Langevin equation (2 1) and integiate over $\hat{\mathbf{n}}$ to obtain the continuity equation,

$$
\frac{\partial}{\partial t} \rho(\mathbf{r}, \varepsilon, t)+\frac{\partial}{\partial \mathbf{r}} \mathbf{j}(\mathbf{r}, \varepsilon, t)+e \mathbf{E}(\mathbf{r}, t) \frac{\partial}{\partial \varepsilon} \mathbf{j}(\mathbf{r}, \varepsilon, t)=0,
$$

for the energy-resolved charge and current densities

$$
\begin{gathered}
\rho(\mathbf{r}, \varepsilon, t)=e \nu(\varepsilon) \mathcal{F}(\mathbf{r}, \varepsilon, t), \\
\mathbf{j}(\mathbf{r}, \varepsilon, t)=\frac{1}{d} \operatorname{ev} \nu(\varepsilon) \mathbf{f}(\mathbf{r}, \varepsilon, t),
\end{gathered}
$$

with $v=\sqrt{2 \varepsilon / m}$ In the zero-frequency limit we may omit the time deirvative in $\mathrm{Eq}$ (2 5)

Multiplication by $\hat{\mathbf{n}}$ followed by integration gives a second relation between $\rho$ and $\mathbf{j}^{22}$

$$
\begin{aligned}
\mathbf{j}(\mathbf{r}, \varepsilon, t)= & -D(\varepsilon) \frac{\partial}{\partial \mathbf{r}} \rho(\mathbf{r}, \varepsilon, t)-\sigma(\varepsilon) \mathbf{E}(\mathbf{r}, t) \\
& \times \frac{\partial}{\partial \varepsilon} \mathcal{F}(\mathbf{r}, \varepsilon, t)+\delta \mathbf{J}(\mathbf{r}, \varepsilon, t),
\end{aligned}
$$



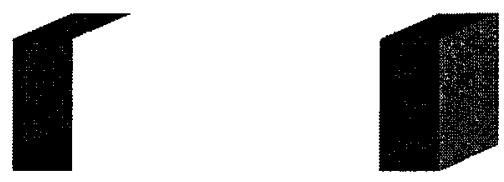

$\stackrel{T \rightarrow}{L}$

FIG. 1. Semiconducting slab (grey) between two metal contacts (black) at $x=0$ and $x=L$. The $(d-1)$-dimensional cross-sectional area is $A$. The current flows from left to right in response to a voltage $V$ applied between the contacts.

a combination of Fick's law and Ohm's law with a fluctuating current source. The conductivity $\sigma(\varepsilon)=e^{2} \nu(\varepsilon) D(\varepsilon)$ is the product of the density of states and the diffusion constant $D(\varepsilon)=v^{2} \tau / d=(2 \varepsilon / m d) \tau(\varepsilon)$. The scattering rate is given by

$$
\frac{1}{\tau(\varepsilon)}=\int \frac{d \hat{\mathbf{n}}^{\prime}}{\Omega} W_{\varepsilon}\left(\hat{\mathbf{n}} \cdot \hat{\mathbf{n}}^{\prime}\right)\left(1-\hat{\mathbf{n}} \cdot \hat{\mathbf{n}}^{\prime}\right) .
$$

The energy-resolved Langevin current

$$
\delta \mathbf{J}(\mathbf{r}, \varepsilon, t)=e \tau(\varepsilon) v \nu(\varepsilon) \int \frac{d \hat{\mathbf{n}}}{\Omega} \hat{\mathbf{n}} \delta J(\mathbf{r}, \hat{\mathbf{n}} \sqrt{2 m \varepsilon}, t)
$$

is correlated as

$$
\begin{aligned}
& \overline{\delta J_{l}(\mathbf{r}, \varepsilon, t) \delta J_{m}\left(\mathbf{r}^{\prime}, \varepsilon^{\prime}, t^{\prime}\right)} \\
& \quad=2 \sigma(\varepsilon) \overline{\mathcal{F}}(\mathbf{r}, \varepsilon, t) \delta_{l m} \delta\left(\mathbf{r}-\mathbf{r}^{\prime}\right) \delta\left(t-t^{\prime}\right) \delta\left(\varepsilon-\varepsilon^{\prime}\right),
\end{aligned}
$$

where we have omitted terms quadratic in $\overline{\mathcal{F}}$.

These kinetic equations should be solved together with the Poisson equation

$$
\kappa \frac{\partial}{\partial \mathbf{r}} \mathbf{E}(\mathbf{r}, t)=\rho(\mathbf{r}, t)-\rho_{\mathrm{eq}},
$$

with $\rho(\mathbf{r}, t)=\int d \varepsilon \rho(\mathbf{r}, \varepsilon, t)$ the integrated charge density, $\kappa$ the dielectric constant, and $\rho_{\mathrm{eq}}$ the mean charge density in equilibrium. The Langevin current $\delta J$ induces fluctuations in $\rho$ and hence in $\mathbf{E}$. The need to take the fluctuations in the electric field into account self-consistently is a severe complication of the problem.

\section{Slab geometry}

We consider the slab geometry of Fig. 1 , consisting of a semiconductor aligned along the $x$ axis with uniform crosssectional area $A$. A nonfluctuating potential difference $V$ is maintained between the metal contacts at $x=0$ and $x=L$, with the current source at $x=0$. The contacts are in equilibrium at temperature $T$. It is convenient to integrate over energy and the coordinates $\mathbf{r}_{\perp}$ perpendicular to the $x$ axis. We define the linear charge density $\rho(x, t)=\int d \mathbf{r}_{\perp} \rho(\mathbf{r}, t)$ and the currents $I(t)=\int d \mathbf{r}_{\perp} \int d \varepsilon j_{\lambda}(\mathbf{r}, \varepsilon, t)$ and $\delta J(x, t)$ $=\int d \mathbf{r}_{\perp} \int d \varepsilon \delta J_{x}(\mathbf{r}, \varepsilon, t)$. The current $I$ is $x$ independent in the zero-frequency limit because of the continuity equation (2.5). We also define the electric-field profile $E(x, t)$
$=A^{-1} \int d \mathbf{r}_{\perp} E_{x}(\mathbf{r}, t)$. The vector $\mathbf{r}_{\perp}$ of transverse coordinates has $d-1$ dimensions. The physically relevant case is $d=3$, but in computer simulations one can consider other values of $d$. For example, in Ref. 12 the case $d=2$ was also studied, corresponding to a hypothetical "flatland.", 23 To compare with the simulations, we will also consider arbitrary $d$.

For any $d$ the fluctuating Ohm-Fick law (2.8) takes the one-dimensional form

$$
\begin{aligned}
I(t)= & -\frac{\partial}{\partial x} \int d \mathbf{r}_{\perp} \int d \varepsilon D(\varepsilon) \rho(\mathbf{r}, \varepsilon, t) \\
& +E(x, t) \int d \mathbf{r}_{\perp} \int d \varepsilon \mathcal{F}(\mathbf{r}, \varepsilon, t) \frac{d}{d \varepsilon} \sigma(\varepsilon)+\delta J(x, t),
\end{aligned}
$$

where we used that the averages of $\mathcal{F}$ and $\mathbf{E}$ depend on $x$ only and neglected terms quadratic in the fluctuations. The Poisson equation (2.12) becomes

$$
\kappa A \frac{\partial}{\partial x} E(x, t)=\rho(x, t)-A \rho_{\mathrm{eq}},
$$

and the correlator (2.11) becomes

$$
\begin{aligned}
& \overline{\delta J(x, t) \delta J\left(x^{\prime}, t^{\prime}\right)} \\
& \quad=2 A \delta\left(t-t^{\prime}\right) \delta\left(x-x^{\prime}\right) \int d \varepsilon \sigma(\varepsilon) \overline{\mathcal{F}}(x, \varepsilon) .
\end{aligned}
$$

Our problem is to compute from Eqs. (2.13)-(2.15) the shotnoise power (1.1).

\section{Energy-independent scattering time}

The Ohm-Fick law (2.13) simplifies in the model of an energy-independent scattering time $\tau(\varepsilon) \equiv \tau$. Then the derivative of the conductivity $d \sigma / d \varepsilon=e \mu \nu(\varepsilon)$ is proportional to the density of states and contains the energy-independent mobility $\mu=e \tau / m$. Equation (2.13) becomes

$$
\begin{aligned}
I(t)= & -\frac{\partial}{\partial x} \int d \mathbf{r}_{\perp} \int d \varepsilon D(\varepsilon) \rho(\mathbf{r}, \varepsilon, t) \\
& +\mu \rho(x, t) E(x, t)+\delta J(x, t) .
\end{aligned}
$$

The drift term now has the same form $\mu \rho E$ as for inelastic scattering. ${ }^{24}$ This simple form does not hold for the more general case of energy-dependent elastic scattering.

\section{SPACE-CHARGE LIMITED CONDUCTION}

For a large voltage drop $V$ between the two metal contacts and a high carrier density $\rho_{c}$ in the contacts, the charge injected into the semiconductor is much higher than the equilibrium charge $\rho_{\text {eq }}$, which can then be neglected. For sufficiently high $V$ and $\rho_{c}$, the system enters the regime of spacecharge limited conduction, ${ }^{25}$ defined by the boundary condition

$$
E(x, t)=0 \quad \text { at } \quad x=0 .
$$

Equation (3.1) states that the space charge $Q=\int_{0}^{L} \rho(x) d x$ in the semiconductor is precisely balanced by the surface 
cha1ge at the current drain The accuracy of this boundary condition at finite $V$ and $\rho_{c}$ is examined in Sec IV E At the diam we have the absorbing boundary condition

$$
\rho(x, t)=0 \quad \text { at } \quad x=L
$$

This is the diffusion appioximation to the condition of zero flux incident fiom the current drain Here we neglect the small theimal contribution to the noise from carrieis that are injected and collected at the drain at kinetic eneigies $\sim k T$, as well as the negligible fiaction $\sim \exp (-e V / k T)$ of carrieis injected from the drain that can overcome the potential balrier

To determine the electric field inside the semiconductor, we proceed as follows The potentral gain $-e \phi(x, t)$ (with $E=-\partial \phi / \partial x)$ dominates over the mitial theimal excitation eneigy of order $k T$ (with Boltzmann's constant $k$ ) almost throughout the whole semiconductor, only close to the curient source (1n a thin boundary layer) this is not the case We can therefore approximate the kinetic eneigy $\varepsilon \approx-e \phi$ and introduce this into $D(\varepsilon)$ and $d \sigma / d \varepsilon$ We assume a power-law energy dependence of the scattering time $\tau$ $=\tau_{0} \varepsilon^{\alpha} \quad$ Then $\quad D(\varepsilon)=\left(2 \tau_{0} / m d\right) \varepsilon^{\alpha+1} \approx-\left(2 \mu_{0} / d\right)$ $(-e)^{\alpha} \phi^{\alpha+1}$ and $d \sigma / d \varepsilon=(2 \alpha+d)\left(\tau_{0} / m d\right) e^{2} \varepsilon^{\alpha} \nu(\varepsilon) \approx$ $-(2 \alpha+d)\left(\mu_{0} / d\right)(-e)^{\alpha+1} \phi^{\alpha} \nu(\varepsilon)$, where we have defined $\mu_{0}=e \tau_{0} / m$ Substituting into Eq (2 13) and using the Poisson equation $-\kappa A \partial^{2} \phi / \partial x^{2}=\rho$, we find the third-order, nonlinear, inhomogeneous differential equation

$$
\begin{gathered}
(2 \alpha+d) \phi^{\alpha} \frac{\partial}{\partial x}\left(\frac{\partial \phi}{\partial x}\right)^{2}-4 \frac{\partial}{\partial x}\left(\phi^{\alpha+1} \frac{\partial^{2} \phi}{\partial x^{2}}\right) \\
=\frac{2 d}{(-e)^{\alpha} \mu_{0} \kappa A}[I(t)-\delta J(x, t)]
\end{gathered}
$$

for the potential profile $\phi(x, t)$

Since the potential difference $V$ between source and diain does not fluctuate, we have the two boundary conditions

$$
\begin{gathered}
\phi(x, t)=0 \quad \text { at } \quad x=0, \\
\phi(x, t)=-V \quad \text { at } \quad x=L
\end{gathered}
$$

Equations (3 1) and (3 2) imply two additional boundary conditions

$$
\begin{aligned}
& \frac{\partial}{\partial x} \phi(x, t)=0 \quad \text { at } \quad x=0, \\
& \frac{\partial^{2}}{\partial x^{2}} \phi(x, t)=0 \quad \text { at } \quad x=L
\end{aligned}
$$

We will now solve this boundary value problem for $\phi$ $=\bar{\phi}+\delta \phi$, first for the mean and then for the fluctuations, in both cases neglecting terms quadratic in $\delta \phi$ The case $\alpha$ $=0$ of an energy-1ndependent scattering time is considered first, in Sec IV The more complicated case of nonzeio $\alpha$ is treated in Sec V

\section{ENERGY-INDEPENDENT SCATTERING TIME}

\section{A. Average profiles}

Fol $\alpha=0$ the averaged equation (3 3) can be integrated once to obtain the second-order differential equation

$$
\left(\frac{d \bar{\phi}}{d x}\right)^{2}-\frac{4}{d} \bar{\phi} \frac{d^{2} \bar{\phi}}{d x^{2}}=\frac{2 \bar{I}}{\mu \kappa A} x
$$

for the mean potential $\bar{\phi}(x)$ In this case of an energyindependent scattering time $\tau(\varepsilon) \equiv \tau$, we may identify $\mu_{0}$ with the mobility $\mu=e \tau / m$ intioduced in Sec II D No integration constant appears in Eq (41), since only then the boundary conditions ( 34 ) and (36) at $x=0$ can be fulfilled simultaneously In Ref 13 the second term on the left-hand side of $\mathrm{Eq}$ (4 1) (the diffusion teim) was neglected relative to the first teim (the drift term) This appioximation is i1gorously justified only in the formal limit $d \rightarrow \infty$ It has the diawback of reducing the order of the equation by one, so that no longer can all boundary conditions be fulfilled $\mathrm{Al}$ though the solution in Ref 13 violates the absoibing boundaly condition ( 37 ), the final result for the shot-noise power turns out to be close to the exact result obtaned here

Before solving this nonlinear differential equation exactly, we discuss two scaling properties that help us along the way Note first that the current $\bar{I}$ can be scaled away by the substitution

$$
\bar{\phi}(x)=-\left(\frac{2 \vec{I}}{\mu \kappa A}\right)^{1 / 2} \chi(x)
$$

Second, each solution $\chi(x)$ of

$$
\left(\frac{d \chi}{d x}\right)^{2}-\frac{4}{d} \chi \frac{d^{2} \chi}{d x^{2}}=x
$$

[the rescaled $\mathrm{Eq}(41)$ ] generates a one-parameter family of solutions $\lambda^{3 / 2} \chi(x / \lambda)$ Thus, if we find a solution that fulfills the three boundaly conditions $\chi(0)=0, \chi^{\prime}(0)=0, \chi^{\prime \prime}(1)$ $=0$ (primes denoting differentiation with iespect to $x$ ), then the potential

$$
\bar{\phi}(x)=-\left(\frac{2 \bar{I} L^{3}}{\mu \kappa A}\right)^{1 / 2} \chi(x / L)
$$

solves Eq (4 1) with boundaty conditions (34), (3 6), and (3 7) The remaining boundary condition (35) determmes the current-voltage characteristic

$$
\bar{I}(V)=\frac{\mu \kappa A}{2 L^{3}}\left(\frac{V}{\chi(1)}\right)^{2}
$$

The quadiatic dependence of $\bar{I}$ on $V$ is the Mott-Gurney law of space-chaige limited conduction ${ }^{26}$

We now constiuct a solution $\chi(x)$ One obvious solution is $\chi_{0}(x)=a_{0} x^{3 / 2}$, with

$$
a_{0}=\frac{2}{3}\left(1-\frac{4}{3 d}\right)^{-1 / 2}
$$


This solution satisfies the boundary conditions at $x=0$, but $\chi_{0}^{\prime \prime}(x) \neq 0$ for any finite $x$ Close to the singular point $x=0$ any solution will appioach $\chi_{0}(x)$ provided that $d>4 / 3$ Let us discuss first this range of $d$, containing the physically relevant dimension $d=3$

We substitute into Eq (4 3) the ansatz

$$
\chi(x)=\sum_{l=0}^{\infty} a_{l} x^{\beta l+3 / 2},
$$

consisting of $\chi_{0}(x)$ times a power selies in $x^{\beta}$, with $\beta$ a positive power to be determined This ansatz proves fruitful since both teims on the left-hand side of Eq (43) give the same powers of $x$, starting with order $x^{1}$ in coincidence with the right hand side Power matching gives Eq (46) for $a_{0}$, and for $l \geqslant 1$ it gives the conditions

$$
\begin{gathered}
\sum_{m=0}^{l} b_{l m} a_{m} a_{l-m}=0 \\
b_{l m}=\frac{9}{4}-\frac{3}{d}+\left(3-\frac{8}{d}\right) m \beta-\left(1+\frac{4}{d}\right) m^{2} \beta^{2}+m l \beta^{2}
\end{gathered}
$$

The relation with $l=1$ is special It determines the power $\beta$,

$$
\beta^{2}+\beta\left(2-\frac{3}{4} d\right)+\frac{3}{2}-\frac{9}{8} d=0
$$

but leaves the coefficient $a_{1}$ as a free parameter [to be determined by demanding that $\chi^{\prime \prime}(1)=0$ ] The positive solution of $\mathrm{Eq}(410)$ is

$$
\beta=\frac{3}{8} d-1+\frac{1}{8} \sqrt{9 d^{2}+24 d-32}
$$

We find $\beta=(\sqrt{13}-1) / 4$ for $d=2$ and $\beta=3 / 2$ for $d=3$ For $l \geqslant 2$ we solve for $a_{l}$ to obtain the iecursion relation

$$
a_{l}=-\frac{\sum_{m=1}^{l-1} b_{l m} a_{m} a_{l-m}}{\left(b_{l l}+b_{l 0}\right) a_{0}}
$$

Interestingly enough, the power series terminates for $d$ $=12 / 5$, and the solution for this dimension is $\chi(x)=x^{3 / 2}$ $-\frac{1}{5} x^{5 / 2}$ For arbitrary dimension $d>4 / 3$, the coefficients $a_{l}$ fall off with $l$, the more rapidly so the latger $d$ is We find numerically that the solution with $\chi^{\prime \prime}(1)=0$ has $a_{1}$ $=03261$ for $d=2$ and $a_{1}=01166$ for $d=3$

For $d<4 / 3$ we substitute into $\mathrm{Eq}$ (43) the ansatz

$$
\chi(x)=\sum_{l=0}^{\infty} c_{l} x^{\gamma l+(3-\gamma) / 2},
$$

with $\gamma=(4-3 d) /(4-d)$ Now the coefficient $c_{0}$ is free Power matching gives, further,

$$
c_{1}=-\frac{d}{4 \gamma(\gamma+1)}
$$

and the recuision ielation
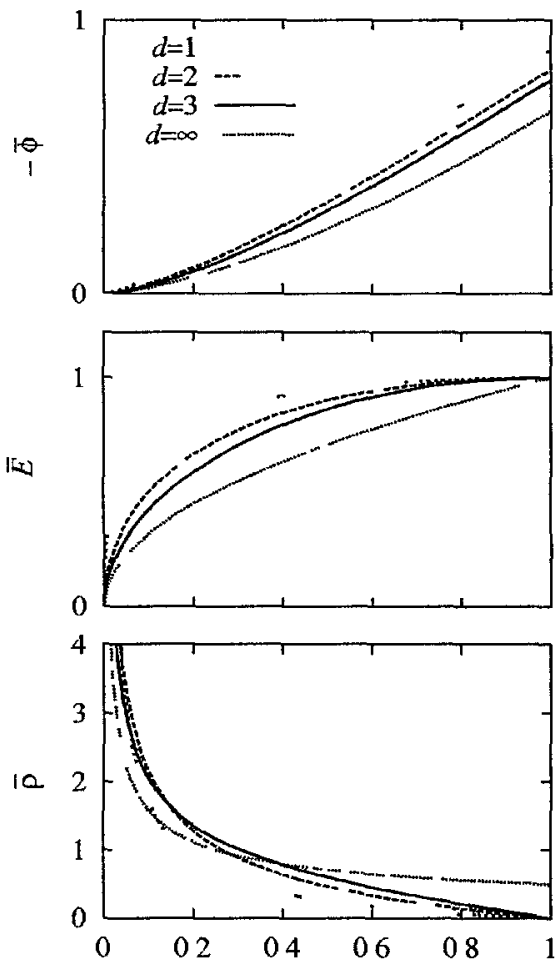

$x / L$

FIG 2 Profile of the mean electrical potential $\bar{\phi}$ [1n units of $\left.\left(2 \vec{I} L^{3} / \mu \kappa A\right)^{1 / 2}\right]$ the electric field $\vec{E}$ [1n units of $\left.(2 \bar{I} L / \mu \kappa A)^{1 / 2}\right]$ and the charge density $\bar{\rho}$ [n units of $(2 \bar{I} \kappa / \mu L A)^{1 / 2}$ ] following from $\mathrm{Eq}(41)$ for different values of $d$ The drift approximation of Ref 13 corresponds to the case $d=\infty$ in this plot

$$
\begin{gathered}
c_{l}=-\frac{\sum_{m=1}^{l-1} d_{l m} c_{m} c_{l-m}}{\left(d_{l l}+d_{l 0}\right) c_{0}}, \\
d_{l m}=\left(\gamma m+\frac{3-\gamma}{2}\right)\left[\gamma(l-m)-\frac{4}{d} \gamma m\right],
\end{gathered}
$$

for coefficients with $l \geqslant 2$ For $d=1$ the solution with $\chi^{\prime \prime}(1)=0$ has $c_{0}=13628$

In Fig 2 the profiles of the potential $\bar{\phi} \propto \chi$, the electric field $\bar{E} \propto \chi^{\prime}$, and the charge density $\bar{\rho} \propto \chi^{\prime \prime}$ are plotted for $d$ $=1,2$, and 3 We also show the result for $d=\infty$, conesponding to the drift approximation of Ref 13 The coefficient $\chi(1)$ appearing in the curient voltage characteristic (45) can be read off from this plot We find $\chi(1)=8 / 9$ for $d=1, \chi(1)=08180$ for $d=2$, and $\chi(1)=07796$ for $d=3$ The limiting value for $d=\infty$ is $\chi(1)=2 / 3$

\section{B Fluctuations}

For the fluctuations it is again convenient to work with the tescaled mean potential (42) We rescale the fluctuations in the same way

$$
\delta \phi(x, t)=-\left(\frac{2 \bar{I}}{\mu \kappa A}\right)^{-1 / 2} \psi(x, t)
$$


We linearize Eq (3 3) with $\alpha=0$ around the mean values and integrate once to obtain the second-order inhomogeneous linear differential equation

$$
\begin{aligned}
\mathcal{L}[\psi] & =-\left(\frac{4}{d} \chi\right) \frac{\partial^{2} \psi}{\partial x^{2}}+\left(2 \frac{d \chi}{d x}\right) \frac{\partial \psi}{\partial x}-\left(\frac{4}{d} \frac{d^{2} \chi}{d x^{2}}\right) \psi \\
& =\int_{0}^{x} d x^{\prime} \frac{\delta I(t)-\delta J\left(x^{\prime}, t\right)}{\bar{I}}
\end{aligned}
$$

The integiation constant vanishes as a consequence of the boundary condition

$$
\psi(x, t)=0 \quad \text { at } \quad x=0
$$

and the iequirement that the fluctuating electric field $\partial \psi / \partial x$ stays finite at $x=0$ [The latter condition actually implies $\partial \psi / \partial x=0$ at $x=0$ ] We will solve Eq $(418)$ with the add1tional condition of a nonfluctuating voltage,

$$
\psi(x, t)=0 \quad \text { at } \quad x=L
$$

The remainıng constraint

$$
\frac{\partial^{2}}{\partial x^{2}} \psi(x, t)=0 \quad \text { at } \quad x=L
$$

(the absorbing boundary condition) will be used later to 1elate $\delta I$ to $\delta J$

We need the Gieen function $G\left(x, x^{\prime}\right)$, satisfying for each $x^{\prime}$ the equation $\mathcal{L}\left[G\left(x, x^{\prime}\right)\right]=\delta\left(x-x^{\prime}\right)$ In view of Eq (43) for the mean potential one recognizes

$$
\psi_{1}(x)=3 \chi(x)-2 x \frac{d}{d x} \chi(x)
$$

as a solution $\mathcal{L}\left[\psi_{1}\right]=0$, which alı eady satısfies $\mathrm{Eq}$ (4 19) Using a standard presci1ption, ${ }^{27}$ we find from $\psi_{1}(x)$ a second, independent, homogeneous solution

$$
\psi_{2}(x)=\psi_{1}(x) \int_{\lambda}^{L} d x^{\prime} \frac{\chi^{d / 2}\left(x^{\prime}\right)}{\psi_{1}^{2}\left(x^{\prime}\right)}
$$

which fulfills $\mathrm{Eq}$ (4 20) The Wronskian is

$$
\psi_{1}(x) \frac{d}{d x} \psi_{2}(x)-\psi_{2}(x) \frac{d}{d x} \psi_{1}(x)=-\chi^{d / 2}(x)
$$

The Green function also contains the factor $-4 \chi / d$ that appears in Eq (4 18) in front of the second-order derivative of $\psi$ We find

$$
\begin{aligned}
G\left(x, x^{\prime}\right)= & \frac{d}{4 \chi^{d / 2+1}\left(x^{\prime}\right)}\left[\Theta\left(x-x^{\prime}\right) \psi_{2}(x) \psi_{1}\left(x^{\prime}\right)\right. \\
& \left.+\Theta\left(x^{\prime}-x\right) \psi_{1}(x) \psi_{2}\left(x^{\prime}\right)\right],
\end{aligned}
$$

where $\Theta(x)=1$ for $x>0$ and $\Theta(x)=0$ for $x<0$

The solution of the inhomogeneous equation (4 18) with boundary conditions (4 19), (4 20) is then

$$
\psi(x, t)=\int_{0}^{L} d x^{\prime} G\left(x, x^{\prime}\right) \int_{0}^{x^{\prime}} d x^{\prime \prime} \frac{\delta I(t)-\delta J\left(x^{\prime \prime}, t\right)}{\bar{I}}
$$

From the extra condition (421) we find

$$
\delta I(t)=\mathcal{C}^{-1} \int_{0}^{L} d x \delta J(x, t) \mathcal{G}(x),
$$

with the definitions

$$
\begin{gathered}
\mathcal{C}=\left(\frac{3}{2} \chi(L)-L^{3 / 2}\right)+\frac{d}{4} \frac{\chi^{d / 2}(L)}{\sqrt{L}} \int_{0}^{L} d x \frac{x \psi_{1}(x)}{\chi^{d / 2+1}(x)} \\
\mathcal{G}(x)=\left(\frac{3 \chi(L)}{2 L}-\sqrt{L}\right)+\frac{d}{4} \frac{\chi^{d / 2}(L)}{\sqrt{L}} \int_{x}^{L} d x^{\prime} \frac{\psi_{1}\left(x^{\prime}\right)}{\chi^{d / 2+1}\left(x^{\prime}\right)}
\end{gathered}
$$

Equation (4 27) is the relation between the fluctuating total current $\delta I$ and the Langevin curnent $\delta I$ that we need to compute the shot-norse power

\section{Shot-noise power}

The shot-noise power is found by substituting Eq (4 27) into Eq (1 1) and invoking the correlator (2 15) for the Langevin curient This iesults in

$$
\begin{gathered}
P=2 \int_{0}^{L} d x\left(\frac{\mathcal{G}(x)}{\mathcal{C}}\right)^{2} \mathcal{H}(x), \\
\mathcal{H}(x)=2 A \int d \varepsilon \sigma(\varepsilon) \overline{\mathcal{F}}(x, \varepsilon)
\end{gathered}
$$

In order to deteimme the mean occupation number $\overline{\mathcal{F}}(x, \varepsilon)$ out of equilibixum, it is conventent to change vat rables from kinetic energy $\varepsilon$ to total energy $u=\varepsilon+e \bar{\phi}(x, t)$ In the new variables $x$ and $u$ we find from the kinetic equations (25) and (28),

$$
\frac{\partial}{\partial x} J(x, u)=0
$$

$$
\bar{J}(x, u)=-\frac{1}{e} \sigma[u-e \bar{\phi}(x)] \frac{\partial}{\partial x} \overline{\mathcal{F}}(x, u)
$$

The derrvatives with respect to $x$ are taken at constant $u$ The solution is

$$
\overline{\mathcal{F}}(x, u)=e \bar{J}(u) \int_{1}^{L} \frac{d x^{\prime}}{\sigma\left[u-e \bar{\phi}\left(x^{\prime}\right)\right]},
$$

where we used the absorbing boundary condition (37) [which implies $\overline{\mathcal{F}}(L, u)=0$ ]

As before [in the derivation of $\mathrm{Eq}$ (3 3) from $\mathrm{Eq}$ (2 13)] we approximate $u-e \bar{\phi}(x) \approx-e \bar{\phi}(x)$ in the algument of $\sigma$ (Th1s is justified because $0<u \leq k T \ll e V)$ Then $\overline{\mathcal{F}}(x, u)$ factorizes into a function of $x$ times a function of $u$, and $\mathrm{Eq}$ (431) gives 


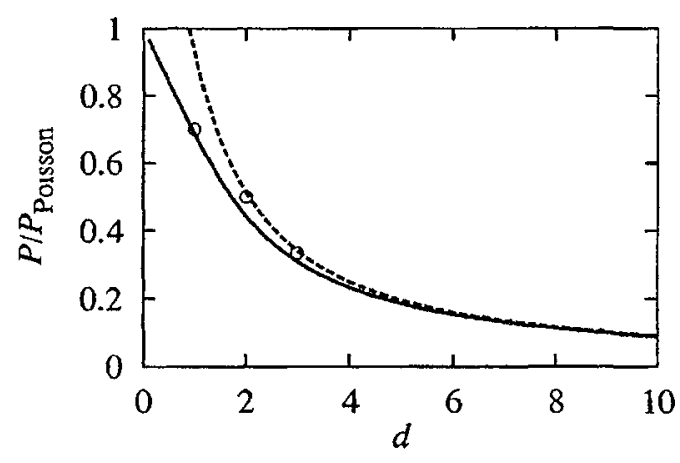

FIG 3 Shot-nolse power $P$ for an energy independent scattering rate as a function of $d$ The exact result (solid curve) is compared with the approximate result (12) (dashed curve) Both curves approach $4 / 5 d$ for $d \rightarrow \infty$ The data points are the results of numer1cal simulations (Ref 12)

$$
\mathcal{H}(x)=2 e \bar{I} \chi^{d / 2}\left(x^{\prime}\right) \int_{x}^{L} d x^{\prime} \chi^{-d / 2}\left(x^{\prime}\right)
$$

where we expressed the result in terms of the rescaled potential $\chi$ In this equation we recognize the Poissonian shotnotse powel $P_{\text {Poisson }}=2 e \bar{I}$

The integrals in the expressions (4 28), (4 29), and (4 35) for $\mathcal{C}, \mathcal{G}$, and $\mathcal{H}$ can be performed with the help of the fact that $\chi$ solves the differential equation (43) In view of this equation,

$$
\begin{gathered}
\chi^{-d / 2}=-\frac{4}{d} \frac{d}{d x}\left(\chi^{1-d / 2} \chi^{\prime \prime}\right), \\
\frac{x \chi^{\prime}}{\chi^{d / 2+1}}=-\frac{2}{d} \frac{d}{d x}\left(\chi^{-d / 2} \chi^{\prime 2}\right), \\
\frac{x \psi_{1}}{\chi^{d / 2+1}}=\frac{4}{d} \frac{d}{d x}\left[\left(x \chi^{\prime 2}-\chi \chi^{\prime}-x \chi \chi^{\prime \prime}\right) \chi^{-d / 2}\right],
\end{gathered}
$$

resultıng in

$$
\begin{gathered}
\mathcal{C}=\frac{1}{2} \chi(L) \\
\mathcal{H}(x)=P_{\text {Poisson }} \frac{4}{d} \chi(x) \chi^{\prime \prime}(x) \\
\mathcal{G}(x)=\frac{1}{\sqrt{L}}\left[3 \chi(x) \chi^{\prime \prime}(x)-\chi^{\prime 2}(x)\right]\left(\frac{\chi(L)}{\chi(x)}\right)^{d / 2}+\frac{3 \chi(L)}{2 L}
\end{gathered}
$$

Our final expression for the shot-noise powel is

$$
P=P_{\text {Polsson }} \frac{32}{d} \frac{1}{\chi^{2}(L)} \int_{0}^{L} d x \mathcal{G}^{2}(x) \chi(x) \frac{d^{2}}{d x^{2}} \chi(x)
$$

The scaling properties of $\chi$ imply that this result does not depend on the length $L$ For $d=1,2$, and 3 it evaluates to

$$
P / P_{\text {Poisson }}= \begin{cases}06857 & \text { for } d=1 \\ 04440 & \text { for } d=2 \\ 03097 & \text { for } d=3\end{cases}
$$

In Fig 3 we plot Eq (4 42) as a function of the dimension $d$ and compare it with the approximate formula (1 2), obtained in Ref 13 by neglecting the diffusion term in Eq (4 1) The exact result (442) is smaller than the approximate result (1 2) by about $10 \%, 15 \%$, and $25 \%$ for $d=3,2$, and 1 , respectively For $d \rightarrow \infty$, the difft approximation that leads to Eq (1 2) becomes stuctly justified, and $P / P_{\text {Poisson }}$ ap proaches $4 / 5 d$ The data points in Fig 3 are the result of the numerical simulation ${ }^{12}$ The agreement with the theory presented here is quite satisfactory, although our findings do not support the conclusion of Ref 12 that $P=\frac{1}{3} P_{\text {Poisson }} 1$ in thiee dimensions

\section{Capacitance fluctuations}

The fluctuations $\delta I(t)$ in the curnent $I(t)$ are due in part to fluctuations in the total charge $Q(t)=\int d x \rho(x, t)$ in the semiconductor The contibution from this soutce to the cur1ent fluctuations is $\delta I_{Q}=(\delta Q / \bar{Q}) \bar{I}$ Fluctuations in the car11er velocities account for the iemaining curient fluctuations $\delta I_{V}=\delta I-\delta I_{Q}$ Since the fluctuations in $Q$ could be measured capacitatively, it is of interest to compute their magnitude separately Because we have assumed that there is no charge present in equilibrium in the semiconductor, $Q(t)$ $=C(t) V$ is diectly piopoitional to the applied voltage $V$ The propoitionality constant $C(t)$ is the fluctuating capac1tance of the semiconductor (The voltage does not fluctuate)

With the Poisson equation (2 14) and the boundary con dition (3 1) we have

$$
C(t)=\frac{\kappa A}{V} E(L, t)
$$

The conelator of the capacitance fluctuations,

$$
P_{C}=2 \int_{-\infty}^{\infty} d t \overline{\delta C(0) \delta C(t)}
$$

1S related to the correlator of $\delta I_{Q}$,

$$
P_{Q}=2 \int_{-\infty}^{\infty} d t \overline{\delta I_{Q}(0) \delta I_{Q}(t)}
$$

by $P_{Q}=\left(\mu \bar{I} V^{2} / 2 \kappa A L\right) P_{C}$ We also define the conelators

$$
P_{V}=2 \int_{-\infty}^{\infty} d t \overline{\delta I_{V}(0) \delta I_{V}(t)}
$$

$$
P_{Q V}=4 \int_{-r}^{\infty} d t \overline{\delta I_{Q}(0) \delta I_{V}(t)}
$$

such that $P=P_{Q}+P_{V}+P_{Q V}$

In view of Eqs (3 3), (4 18) and the boundary conditions (3 5), (37), one obtains $\bar{E}(L)$ and $\delta E(L, t)$ as a function of $\delta I$ and $\delta J$, and hence 


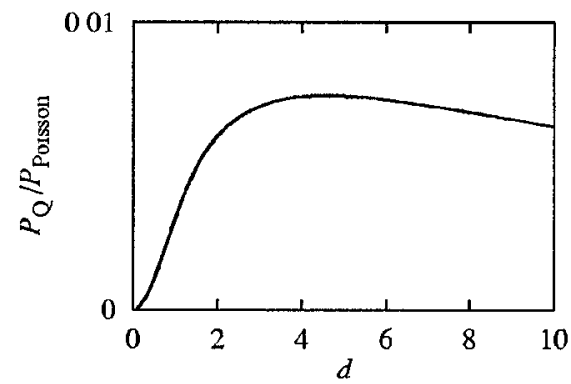

FIG 4 Contribution $P_{Q}$ from charge fluctuations to the shotnoise power $P$ The correlator $P_{C}$ of the capacitance fluctuations is related to $P_{Q}$ by $P_{C}=\left(4 e \kappa A L / \mu V^{2}\right) P_{Q} / P_{\text {Poisson }}$

$$
\begin{aligned}
& \delta I_{N}(t)=\frac{1}{2}\left(\delta I(t)-\int_{0}^{L} \frac{d x}{L} \delta J(x, t)\right), \\
& \delta I_{V}(t)=\frac{1}{2}\left(\delta I(t)+\int_{0}^{L} \frac{d x}{L} \delta J(x, t)\right)
\end{aligned}
$$

With the help of Eq (427) we find

$$
\begin{gathered}
P_{C}=\frac{1}{4}\left(P+P_{J}-2 P_{I J}\right), \\
P_{V}=\frac{1}{4}\left(P+P_{J}+2 P_{I J}\right), \\
P_{C V}=\frac{1}{2}\left(P-P_{J}\right), \\
P_{I J}=\frac{16}{d \chi(L)} P_{\text {Poisson }} \int_{0}^{L} \frac{d x}{L} \mathcal{G}(x) \chi(x) \frac{d^{2}}{d x^{2}} \chi(x), \\
P_{J}=\frac{8}{d} P_{\text {Poisson }} \int_{0}^{L} \frac{d x}{L^{2}} \chi(x) \frac{d^{2}}{d x^{2}} \chi(x)
\end{gathered}
$$

The integrals can be evaluated by using that $\chi(x)$ solves $\mathrm{Eq}$ (43), with the result

$$
\begin{gathered}
P_{I J}=4 \frac{48 L^{-3 / 2} \chi(L)-d-36}{(d+4)(1-5 d)} P_{\text {Poisson }}, \\
P_{J}=4 \frac{2 L^{-3 / 2} \chi(L)-1}{d+4} P_{\text {Poisson }}
\end{gathered}
$$

In Fig 4 the correlator of the capacitance fluctuations is plotted as a function of $d$ For $d=3$ we find $P_{C}$ $=00284 e \kappa A L / \mu V^{2}$ The corresponding contribution $P_{Q}$ $=00071 P_{\text {Poisson }} 1$ is relatively small, being less than $3 \%$ of the contribution from the velocity fluctuations $P_{V}$ $=03076 P_{\text {Poisson }}$ (Incidentally, we find that charge and velocity fluctuations are anticorrelated, $P_{Q V}=$ $\left.-00049 P_{\text {Poisson }}\right)$ Our calculation thus confirms the numer1 cal finding of Ref 12 , that the charge fluctuations are strongly suppressed as a result of Coulomb repulsion However, we do not find the exact cancellation of $P_{Q}$ and $P_{Q V}$ surmised in that paper

\section{E. Effects of a finite voltage and carrier density}

For comparison with realistic systems and with computer simulations, one has to account for a finite voltage $V$ and a finite carrier density $\rho_{c}$ in the metal contacts The density $\rho_{c}$ is the charge density at the semiconducting side of the interface with the metal contact It depends on temperature according to ${ }^{26} \rho_{c}=2 e\left(m k T / 2 \pi \hbar^{2}\right)^{3 / 2} \exp (-W / k T)$, where $W$ is the work function of the interface The relevant parameters are the ratios $L_{c} / L$ and $L_{s} / L$, with $L_{c}=\left(\kappa k T / e \rho_{c}\right)^{1 / 2}$ the Debye screening length in the contact and $L_{s}=\left(\kappa V / \rho_{c}\right)^{1 / 2}$ the screening length in the semiconductor The theory of space-charge limited conduction applies to the regime $L$ $\gg L_{1} \gg L_{c}$ (or $k T \ll e V$ and $\rho_{c} \gg k V / L^{2}$ - the combination $\kappa V / L^{2}$ characterizing the mean charge density in the semiconductor) In this section we will show that, within this regime, the effects of a finite voltage and carrier density are lestricted to a narrow boundary layer near the current source We will examine the deviations from the boundary condition (31) and compare with the numerical simulations ${ }^{12}$

To investigate the accuracy of the boundary condition (3 1), we start from the more fundamental condition of thermal equilibrium,

$$
\bar{\rho}(x, \varepsilon)=\frac{A \rho_{c} \nu(\varepsilon) \exp (-\varepsilon / k T)}{\int_{0}^{\infty} d \varepsilon^{\prime} \nu\left(\varepsilon^{\prime}\right) \exp \left(-\varepsilon^{\prime} / k T\right)} \text { at } x=0
$$

We keep the absoibing boundary condition $\bar{\rho}(L, \varepsilon)=0$ at the current drain, since thermally excited carriers injected from the contact at $x=L$ make only a small contribution to the current when $e V \gg k T$ To simplify the problem, we assume that all carriers at the current souıce have the same kinetic energy $\frac{1}{2} d k T$, in essence replacing the Boltzmann factor $\exp (-\varepsilon / k T)$ in Eq (458) by a delta function at $\varepsilon=(d / 2) k T$ We restrict ourselves to the physically relevant case $d=3$ and substitute $\varepsilon=\frac{3}{2} k T-e \bar{\phi}(x)$ in the argument of $D(\varepsilon)$ in Eq (2 16) Repeating the steps that resulted in Eq (4 1), we arrive at the differential equation

$$
\left(\frac{d \bar{\phi}}{d x}\right)^{2}-\left(\frac{4}{3} \bar{\phi}-2 \frac{k T}{e}\right) \frac{d^{2} \bar{\phi}}{d x^{2}}=\frac{2 \bar{I}}{\mu \kappa A}(x-\xi)
$$

In comparison to $\mathrm{Eq}(41)$, an integration constant $\xi$ appears now on the inght-hand side This constant and the current $\bar{I}$ have to be determined from the foul boundary conditions $\bar{\phi}(0)=0, \kappa \bar{\phi}^{\prime \prime}(0)=-\rho_{c}, \bar{\phi}(L)=-V$, and $\bar{\phi}^{\prime \prime}(L)=0$

We have integrated $\mathrm{Eq}$ (459) numerically In $\mathrm{F}_{1} \mathrm{~g} 5$ we show the electric field for $d=3$ and parameters as in the simulations of Ref 12 , corresponding to $L / L_{r}=489$ and $\left(L_{s} / L_{c}\right)^{2}=e V / k T$ ranging between 40 and 300 We find excellent agreement, the better so the larger $e V / k T$ is, without any adjustable parameter

The boundary condition (3 1) of zeio electric field at the current source assumes that the surface charge in the current drain is fully screened by the space charge in the semiconductor With increasing $e V / k T$ for fixed $L / L$, one observes in Fig 5 a transition from oveiscreening $(\vec{E}=0$ at a point inside the semiconductor) to underscreening ( $\bar{E}$ extrapolates to zero at a point inside the metal contact) We can approximate $\bar{E}(x)=-\bar{\phi}_{0}^{\prime}(x-\xi)$, where $\bar{\phi}_{0}$ solves Eq (41) with the boundary conditions of space-charge limited conduction 

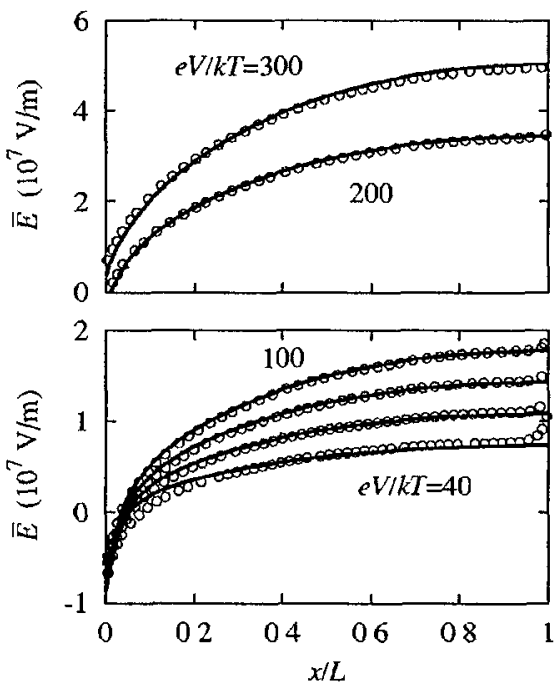

FIG 5 Electric-field profiles for $e V / k T=40,60,80,100,200$, and 300 , at parameter values $d=3, T=300 \mathrm{~K}, \rho_{c} / e=10^{24} \mathrm{~m}^{3}$, $L=200 \mathrm{~nm}$, and $\kappa=117 \kappa_{0}$ (with $\kappa_{0}$ the dielectric constant of vacuum) The solid curves follow from $\mathrm{Eq}$ (459) The data points are the result of numerical simulations (Ref 28) There are no fit ting parameters in this comparison

This is an excellent approximation for $e V / k T=200(\xi / L$ $=002)$ and $e V / k T=300(\xi / L=-0004)$, piactically indistinguishable from the curves in Fig 5 (top panel)

To demonstrate analytically that space-chaige Iimited conduction is chatacterized by the conditions $L \gg L_{\mathrm{s}} \gg L_{\mathrm{c}}$, we will now compute the width of the boundary layer and show that it becomes $\ll L$ in this regime We need to distınguish between two length scales $\xi$ and $\xi^{\prime}$ to fully character1ze the boundaty layer The length $\xi$ deteimines the shift in the asymptotic solution

$$
\bar{\phi}_{\mathrm{asym}}(x)=\bar{\phi}_{0}(x-\xi)+3 k T / 2 e,
$$

while the size $\xi^{\prime}$ charactelizes the range $0 \leqslant x \leqslant \xi^{\prime}$ where the exact solution $\bar{\phi}(x)$ deviates substantially from $\bar{\phi}_{\text {asym }}(x)$

The values of $\xi$ and $\xi^{\prime}$ are found by comparing Eq (4 60) with the Taylor seires

$$
\bar{\phi}(x)=-E_{0} x-\frac{\rho_{c}}{\kappa} \frac{x^{2}}{2}+\phi_{3} \frac{x^{3}}{6}+O\left(x^{4}\right)
$$

The coefficients in the Taylor sei ies are determined from Eq (4 59),

$$
\begin{aligned}
& E_{0}^{2}-2 \frac{k T}{e} \frac{\rho_{c}}{\kappa}=-\xi \frac{2 \bar{I}}{\mu \kappa A}, \\
& \frac{2}{3} E_{0} \frac{\rho_{c}}{\kappa}+2 \frac{k T}{e} \phi_{3}=\frac{2 \bar{I}}{\mu \kappa A},
\end{aligned}
$$

where $2 \bar{I} / \mu \kappa A \approx V^{2} / L^{3}$ up to a coefficient of order unity [cf $\mathrm{Eq}$ (4 5)]

We match the two functions (460) and (4 61) at $x=\xi^{\prime}$, demanding that potential and electric field are contınuous at $x=\xi^{\prime}$ These two conditions deteimine $\xi$ and $\xi^{\prime}$ Within the regime $L \gg L_{s} \gg L_{c}$ we find two subregimes, depending on the relative magnitude of $L, / L$ and $\left(L_{s} / L\right)^{4}$ Overscreening occurs when $L_{c} / L \gg\left(L_{s} / L\right)^{4}$ Then $E_{0} \approx-\left(2 k T \rho_{c} / e \kappa\right)^{1 / 2}$, $\phi_{3} \approx(2 e / 9 k T)^{1 / 2}\left(\rho_{c} / \kappa\right)^{3 / 2}$, and $\xi \approx \xi^{\prime}=O\left(L_{c}\right)$ The difference $\xi^{\prime}-\xi=O\left(L_{s}^{4} / L^{3}\right) \ll \xi$ At the matching point, $\bar{\phi}$ $=O(k T / e), \quad \bar{E}=O\left(V^{2} \kappa / \rho_{c} L^{3}\right), \quad$ and $\bar{\rho}=O\left(\rho_{c}\right) \quad$ Underscreening occurs when $L_{c} / L \ll\left(L_{s} / L\right)^{4}$ Then $E_{0}$ $=O\left(V^{2} \kappa / \rho L^{3}\right) \ll V / L, \quad \phi_{3}=O\left(\rho_{c}^{3} L^{3} / \kappa^{3} V^{2}\right), \quad \xi=$ - $O\left(L_{s}^{4} / L^{3}\right)$, and $\xi^{\prime}=O\left(L_{s}^{4} / L^{3}\right)$ At the matching point, $\bar{\phi}$ $=O\left(V^{4} \kappa^{3} / \rho_{\mathrm{c}}^{3} L^{6}\right), \bar{E}=O\left(E_{0}\right)$, and $\bar{\rho}=O\left(\rho_{c}\right)$ In between these two subregimes, when $L_{s}^{4} / L^{3} L_{c}$ is of order unity, $\xi^{\prime}$ vanishes and $\bar{\phi}_{\text {asym }}(x)$ becomes an exact solution of Eq (4 59), which also fulfills all boundary conditions In the same range, $\xi$ changes sign from positive to negative values

We conclude that the width of the boundary layer is of order $\max \left(L_{c}, L_{s}^{4} / L^{3}\right)$ At the matching pount, $\bar{E} \ll V / L$ The boundary condition $(31)$, used to calculate the shot-noise power $P$, ignores the boundary layer This is justified because $P$ is a bulk property We estimate the contribution to $P / P_{\text {Poisson }}$ coming fiom the boundaly layel to be of order $\max \left(L_{c} / L,\left(L_{s} / L\right)^{4}\right)$ (possibly to some positive power), hence to be $\ll 1$ in the legime of space-charge limited conduction

\section{ENERGY-DEPENDENT SCATTERING TIME}

We consider now an energy-dependent scattering time We restrict ouiselves to $d=3$ and assume a power-law dependence $\tau(\varepsilon)=\tau_{0} \varepsilon^{\alpha}$ The energy-dependence of the rate $1 / \tau$ is governed by the product of the scattering cross section and the density of states For shoit-1ange impuity scatterm the cioss section is eneigy independent, hence $\alpha=-1 / 2$ This applies to uncharged impuities in semiconductors For scattering by a Coulomb potentral, the cross section is $\sigma \varepsilon^{-2}$, hence $\alpha=3 / 2$ This applies to scattering by charged impurities in semiconductors ${ }^{29}$ The case $\alpha=0$ considered so fai lies between these two extremes ${ }^{30}$ We have found an exact analytical solution for the case of short-1ange scattering, to be presented below The case of long-range impurity scattering remains an open problem, as discussed at the end of this section

Fol shoit-range impunty scattering, the technical steps are similar to those of Sec IV We first determine the mean potentral $\bar{\phi}(x)$ The scaling properties of Eq (3 3) are exploited by introducing the rescaled potential $\chi(x)$, with

$$
\bar{\phi}(x)=-\left(\frac{3 e^{1 / 2} L^{3} \bar{I}}{2 \mu_{0} \kappa A}\right)^{2 / 3} \chi(x / L)
$$

In this way we eliminate the dependence on the curtent $\bar{I}$ and the length of the conductor $L$ The rescaled potentral fulfills the differential equation

$$
\frac{1}{2} \chi^{-1 / 2} \frac{d \chi}{d x} \frac{d^{2} \chi}{d x^{2}}-\chi^{1 / 2} \frac{d^{3} \chi}{d x^{3}}=1
$$

with boundaty conditions $\chi(0)=0, \chi^{\prime}(0)=0$, and $\chi^{\prime \prime}(1)$ $=0$

We substitute 


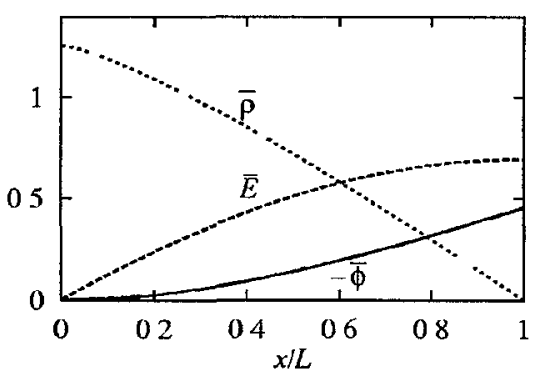

FIG 6 Profile of the mean electrical potential $\bar{\phi}$ [in units of $\left.L^{2}\left(3 e^{1 / 2} \bar{I} / 2 \mu_{0} \kappa A\right)^{2 / 3}\right]$, the electric field $\bar{E}$ [1n units of $\left.L\left(3 e^{1 / 2} \bar{I} / 2 \mu_{0} \kappa A\right)^{2 / 3}\right]$, and the charge density $\bar{\rho}[$ in units of $\left.\left(3 e^{1 / 2} \bar{I} K^{1 / 2} / 2 \mu_{0} A\right)^{2 / 3}\right]$ for a three-dimensional conductor with shortrange impurity scattering, computed from Eq $(52)$

$$
\chi(x)=\sum_{l=0}^{\infty} g_{l} x^{\eta l+2}
$$

into $\mathrm{Eq}$ (52) Power matchıng gives in the first order $g_{0}$ $=2^{-2 / 3}$ The second order leaves $g_{1}$ as a free coefficient, but fixes the power $\eta=(\sqrt{13}-1) / 2$ The coefficients $g_{l}$ for $l$ $\geqslant 2$ are then determined recursively as a function of $g_{1}$ From the condition $\chi^{\prime \prime}(1)=0$, we obtain $g_{1}=-01808$ The resulting series expansion converges rapidly, with the coefficient $g_{12}$ already of order $10^{-12}$

The averaged potential and its first and second derivative are plotted in Fig 6 The electric field $\propto \chi^{\prime}(x)$ increases now linearly at the current source, hence the charge density ${ }^{\alpha} \chi^{\prime \prime}(x)$ remains finite there The current-voltage characteristic is

$$
\bar{I}=\frac{2 \mu_{0} \kappa A}{3 e^{1 / 2} L^{3}}\left(\frac{V}{\chi(1)}\right)^{3 / 2},
$$

with $\chi(1)=04559$ This is a slower increase of $I$ with $V$ than the quadratic increase (45) in systems with energyindependent scattering

The rescaled fluctuations $\psi(x, t)$, introduced by

$$
\delta \phi(x, t)=-\left(\frac{3 e^{1 / 2} L^{3} \bar{I}}{2 \mu_{0} \kappa A}\right)^{2 / 3} \psi(x / L, t)
$$

fulfill the linear differential equation

$$
\begin{aligned}
\mathcal{L}[\psi]= & -\chi^{1 / 2} \frac{\partial^{3} \psi}{\partial x^{3}}+\frac{1}{2} \frac{\chi^{\prime}}{\chi^{1 / 2}} \frac{\partial^{2} \psi}{\partial x^{2}}+\frac{1}{2} \frac{\chi^{\prime \prime}}{\chi^{1 / 2}} \frac{\partial \psi}{\partial x} \\
& -\frac{1}{4}\left(\frac{\chi^{\prime} \chi^{\prime \prime}}{\chi^{3 / 2}}+2 \frac{\chi^{\prime \prime \prime}}{\chi^{1 / 2}}\right) \psi \\
= & \frac{\delta I(t)-\delta J(x, t)}{\bar{I}}
\end{aligned}
$$

The solution of the inhomogeneous equation is found with the help of the three independent solutions of the homogeneous equation $\mathcal{L}[\psi]=0$,

$$
\psi_{1}(x)=\frac{d}{d x} \chi(x)
$$

$$
\begin{gathered}
\psi_{2}(x)=\chi(x)-\frac{x}{2} \frac{d}{d x} \chi(x), \\
\psi_{3}(x)=\psi_{1}(x) \int_{\lambda}^{1} d x^{\prime} \frac{\chi^{1 / 2}\left(x^{\prime}\right) \psi_{2}\left(x^{\prime}\right)}{\mathcal{W}^{2}\left(x^{\prime}\right)} \\
-\psi_{2}(x) \int_{x}^{1} d x^{\prime} \frac{\chi^{1 / 2}\left(x^{\prime}\right) \psi_{1}\left(x^{\prime}\right)}{\mathcal{W}^{2}\left(x^{\prime}\right)},
\end{gathered}
$$

where we have defined

$$
\mathcal{W}(x)=\psi_{1}(x) \psi_{2}^{\prime}(x)-\psi_{1}^{\prime}(x) \psi_{2}(x)
$$

The special solution which fulfills $\psi(0, t)=\psi^{\prime}(0, t)=\psi(1, t)$ $=0$ is

$$
\begin{aligned}
\psi(x, t)= & \int_{0}^{1} d x^{\prime} \frac{\chi^{1 / 2}\left(x^{\prime}\right)}{\mathcal{W}^{2}\left(x^{\prime}\right)}\left[\Theta\left(x-x^{\prime}\right) \psi_{1}(x) \psi_{2}\left(x^{\prime}\right)\right. \\
& \left.+\Theta\left(x^{\prime}-x\right) \psi_{1}\left(x^{\prime}\right) \psi_{2}(x)-\frac{\psi_{1}(1)}{\psi_{2}(1)} \psi_{2}(x) \psi_{2}\left(x^{\prime}\right)\right] \\
& \times \int_{0}^{x^{\prime}} d x^{\prime \prime} \frac{\delta I(t)-\delta J\left(x^{\prime \prime}, t\right)}{\bar{I}} \frac{\mathcal{W}\left(x^{\prime \prime}\right)}{\chi\left(x^{\prime \prime}\right)}
\end{aligned}
$$

The condition $\psi^{\prime \prime}(1, t)=0$ relates the fluctuating curtent $\delta I$ to the Langevin current $\delta J$ The resulting expiession is again of the form (427), with now

$$
\begin{gathered}
\mathcal{C}=\int_{0}^{1} d x \mathcal{G}(x), \\
\mathcal{G}(x)=\frac{\mathcal{W}(x)}{\chi(x)}\left(2+\frac{\chi^{\prime 2}(1)}{\psi_{2}(1)} \int_{x}^{1} d x^{\prime} \frac{\chi^{1 / 2}\left(x^{\prime}\right) \psi_{2}\left(x^{\prime}\right)}{\mathcal{W}^{2}\left(x^{\prime}\right)}\right)
\end{gathered}
$$

The shot-noise power is given by $\mathrm{Eq}$ (430) with $\mathcal{H}(x)$ as defined in Eq (4 31) and the mean occupation number $\overline{\mathcal{F}}$ still given by $\mathrm{Eq}$ (4 34) Instead of $\mathrm{Eq}$ (4 35) we now have

$$
\begin{aligned}
\mathcal{H}(x) & =2 e \bar{I} \chi(x) \int_{x}^{1} d x^{\prime} \frac{1}{\chi\left(x^{\prime}\right)} \\
& =P_{\text {Poisson }} \chi^{1 / 2}(x) \chi^{\prime \prime}(x),
\end{aligned}
$$

where we integrated with the help of Eq (52) and used $\chi^{\prime \prime}(1)=0$

Collecting tesults, we obtain the shot-noise suppression factor

$$
P / P_{\text {Poisson }}=03777 \text {, }
$$

which is about $20 \%$ larger than the result obtained in Sec IV for an energy-independent scattering time in thiee dimensions Equation (515) can be compared with the $\alpha$-dependent iesult in the dift approximation

$$
P / P_{\text {Polsson }}=\frac{6(\alpha-1)(\alpha+2)\left(16 \alpha^{2}+36 \alpha-157\right)}{5(2 \alpha-5)(8 \alpha-17)(13+8 \alpha)}
$$


For $\alpha=-1 / 2$ the drift approximation gives $P$ $=04071 P_{\text {Porsson }}$, about $10 \%$ larger than the exact result (5 15$)$

We now turn bilefly to the case of long-range impuity scattering The kinetic equation (3 3), on which our analysis is based, predicts a logarithmically diverging electic field $\alpha-\ln ^{1 / 3} x$ at the current sounce for $\alpha=1$ In the range $\alpha$ $>1$, which includes the case $\alpha=3 / 2$ of scatten ing by charged impunties, we could not determine the low- $x$ behavior [A behavio1 $\phi \propto C x^{\beta}$ is ruled out because Eq (33) cannot be satisfied with a real coefficient $C]$ In the drift appioximation, the shot-noise power (5 16) vanishes as $\alpha \rightarrow 1$ Presumably, a nonzeio answel for $P$ would follow for $\alpha \geqslant 1$ if the nonzero thermal energy and finite charge density at the cunent source is accounted for This remains an open problem

\section{DISCUSSION}

We have computed the shot-noise power in a nondegenerate diffusive semiconductor, in the regime of space-charge limited conduction, for two types of elastic impurity scattering In thiee-dimensional systems the shot-norse suppression factor $P / P_{\text {Poisson }}$ is close to $1 / 3$ both for the case of an energy-1ndependent scatteing 1 ate $\left(P / P_{\text {Porsson }}=03097\right)$ and for the case of shoit-1ange scattering by uncharged impurities $\left(P / P_{\text {Poisson }}=03777\right)$ (The latter case also applies to quas1elastic scattering by acoustic phonons, discussed below ) Our results are in good agreement with the numerical simulations for energy-independent scattering by González et al ${ }^{12}$ The results in the dift approximation ${ }^{13}$ are about $10 \%$ laiger We found that capacitance fluctuations are strongly suppressed by the long-range Coulomb interaction We discussed the effects of a nonzeio thermal excitation energy and a finite carrier density in the current source and determined the regime $L \gg L_{s} \gg L_{c}$ for space-charge limited conduction $\left(L_{s}\right.$ and $L_{c}$ being the screening lengths in the semiconductor and current source, respectively) Two subregimes of overscreening and underscreening were identıfied, again in quantitatıve agreement with the numerical simulations 12

Let us discuss the conditions for experimental obseivabil ity We have neglected inelastic-scattering events These dive the gas of charge carriers towards local thermal equilibium and result in a suppiession of the shot noise down to thermal noise, $P=8 k T d \bar{I} / d V^{13}$ Inelastic scattering by opt1cal phonons can be neglected for voltages $V<k T_{D} / e$, with $T_{D}$ the Debye temperature Scattering by acoustic phonons is quasielastic as long as the sound velocity $v_{s}$ is much smaller than the typical election velocity $v \approx(\mathrm{eV} / \mathrm{m})^{1 / 2}$ For large enough temperatuses $T \gg m v v_{s} / k$, the elastic-scattering time $\tau \propto \epsilon^{-1 / 2}$ depends on enetgy in the same way as for shortrange impurity scattering ${ }^{31}$

All requirements appear to be realistic for a semiconducting sample with a sufficiently low carrier density The electron gas is degenerate even at quite low temperatures (a few Kelvin) Short-1ange election-electron scattering is rare due to the diluteness of the caniers Scatteing by phonons is piedominantly elastic If the dopant (charged impurities) is sufficiently dilute, the impurity scattering is piedominantly shoit langed Under these conditions we would expect the shot-noise power to be about one-third of the Poisson value

\section{ACKNOWLEDGMENTS}

We are indebted to $\mathrm{J} M \mathrm{~J}$ van Leeuwen for showing us how to solve the nonlineal differential equation (41) We thank $\mathrm{T}$ González for permission to use the data shown in Fig 5 Discussions with O M Bulashenko, A V Khaetsk11, K E Nagaev, and W van Saatloos are gratefully acknowledged This work was supported by the European Community (Plogiam for the Tiainıng and Mobility of Reseaıchers) and by the Dutch Science Foundation NWO/FOM
${ }^{1}$ B B Kadomtsev, Zh Eksp Teor Fiz 32, 943 (1957) [Sov Phys JETP 5, 771 (1957)]

${ }^{2}$ Sh M Kogan and A Ya Shul'man, Zh Éksp Teor Fiz 56, 862 (1969) [Sov Phys JETP 29, 467 (1969)]

${ }^{3}$ S V Gantsevich, V L Gurevich, and R Katilus, Zh Eksp Teor F1z 57, 503 (1969) [Sov Phys JETP 30, 276 (1970)]

${ }^{4}$ Sh Kogan, Electronic Notse and Fluctuations in Solıds (Cambridge University, Cambridge, England, 1996)

${ }^{5} \mathrm{M} \mathrm{J}$ M de Jong and C W J Beenakker, in Mesoscopic Electron Transport, Vol 345 of NATO Advances Studies Institute, Series E Applied Sciences, edited by L L Sohn, L P Kouwenhoven, and G Schon (Kluwer, Dordrecht, 1997)

${ }^{6} \mathrm{C}$ W J Beenakker and M Buttıker, Phys Rev B 46, 1889 (1992)

${ }^{7} \mathrm{~K}$ E Nagaev, Phys Lett A 169, 103 (1992)

${ }^{8} \mathrm{~F}$ Liefrink, J I Dijkhuis, M J M de Jong, L W Molenkamp, and $H$ van Houten, Phys Rev B 49, 14066 (1994)

${ }^{9}$ A H Steınbach, J M Martınıs, and M H Devoret, Phys Rev Lett 76, 3806 (1996)

${ }^{10} \mathrm{R}$ J Schoelkopf, P J Burke, A A Kozhevnikov, D E Prober, and M J Rooks, Phys Rev Lett 78, 3370 (1997)
${ }^{11} \mathrm{M}$ Henry, S Oberholzer, C Strunk, and C Schonenberger, Phys Rev B 59, 2871 (1999)

${ }^{12} \mathrm{~T}$ González, C González, J Mateos, D Pardo, L Reggıanı, O M Bulashenko, and J M Rub1, Phys Rev Lett 80, 2901 (1998), T Gonzalez, J Mateos, D Pardo, O M Bulashenko, and L Reggianı, Phys Rev B 60, 2670 (1999)

${ }^{13}$ C W J Beenakker, Phys Rev Lett 82, 2761 (1999)

${ }^{14} \mathrm{~K}$ E Nagaev, cond-mat/9812357 (unpublished)

${ }^{15} \mathrm{M}$ J M de Jong and C W J Beenakker, Phys Rev B 51, 16 867 (1995), Physica A 230, 219 (1996)

${ }^{16}$ K E Nagaev, Phys Rev B 52, 4740 (1995), 57, 4628 (1998)

${ }^{17}$ V I Kozub and A M Rudin, Phys Rev B 52, 7853 (1995)

${ }^{18} \mathrm{~V}$ L Gurevich and A M Rudin, Phys Rev B 53, 10078 (1995), P1s'ma Zh Eksp F1z 62, 13 (1995) [JETP Lett 62, 12 (1995)]

${ }^{19} \mathrm{Y}$ Naveh, D V Averin, and K K Likharev, Phys Rev Lett 79, 3482 (1997), Phys Rev B 58, 15371 (1998), 59, 2848 (1999)

${ }^{20} \mathrm{Y}$ Naveh, Phys Rev B 58, R 13387 (1998), cond mat/9806348 (unpublished)

${ }^{21}$ E V Sukhorukov and D Loss, Phys Rev Lett 80, 4959 (1998), cond-mat/9809239 (unpublished)

${ }^{22}$ The term $\tau \partial \mathbf{j} / \partial t$ is omitted from Eq (28) because it contributes 
only to the next higher order in the multipole expansion

${ }^{23}$ A physical realization of the case $d=2$ would be a layered material in which each layer contans a two-dimensional electron gas A single layer would not suffice because the Poisson equation would then remain three dimensional and cannot be reduced to the form (2 14)

${ }^{24}$ In the case of inelastic scattering, the drift term $\mu \rho E$ follows, regardless of the energy dependence of the scatterng rate, from the equilibrium condition $\mathcal{F}=-k T \partial \mathcal{F} / \partial \varepsilon$ This condition does not hold when all scattering is elastic

${ }^{25} \mathrm{M}$ A Lampert and P Mark, Current Injection in Solids (Academic, New York, 1970)

${ }^{26}$ N F Mott and R W Gurney, Electronic Processes in Ionic Crystals (Clarendon, Oxford, 1940) The Mott-Gurney law I $\propto V^{2}$ (also known as Ch1ld's law) assumes local equilbrium and hence requires inelastic scattering Here we find the same quadratıc $I$ - $V$ characteristic, but only if the elastic-scattering ume is energy independent (cf Sec V)

${ }^{27} \mathrm{E}$ L Ince, Ordinary Differential Equattons (Dover, New York, 1956), Sec 522

${ }^{28} \mathrm{~T}$ González, J Mateos, D Pardo, O M Bulashenko, and L Reggianı (private communication)

${ }^{29} \mathrm{D}$ Chattopadhyay and $\mathrm{H}$ Queisser, Rev Mod Phys 53, 745 (1981)

${ }^{30}$ Formally, one can also consider the case $\alpha<-1 / 2$ Nagaev (Ref 14) has shown that full shot noise, $P=P_{\text {Poisson }}$, follows for $\alpha$ $=-3 / 2$

${ }^{31}$ V F Gantmakher and Y B Levinson, Carrter Scattering in Met als and Serniconductors (North-Holland, Amsterdam, 1987) 